\title{
THE UNIFORM ASYMPTOTIC EXPANSION OF A CLASS OF INTEGRALS RELATED TO CUMULATIVE DISTRIBUTION FUNCTIONS*
}

\author{
N. M. TEMME $\dagger$
}

\begin{abstract}
An asymptotic expansion is given for a class of integrals for large values of a parameter, which corresponds with the degrees of freedom in a certain type of cumulative distribution functions. The expansion is uniform with respect to a variable related to the random variable of the distribution functions. Special cases include the chi-square distribution and the $F$-distribution.
\end{abstract}

1. Introduction. We consider integrals of the type

$$
F_{a}(\eta)=\left(\frac{a}{2 \pi}\right)^{1 / 2} \int_{-\infty}^{\eta} e^{-a \zeta^{2} / 2} f_{a}(\zeta) d \zeta
$$

for large values of the positive parameter $a$. The independent variable $\eta$ ranges over an unbounded domain

$$
H_{\delta}=\{\eta=x+i y|x \in \mathbb{R},| y \mid<\delta\},
$$

where $\delta>0 ; \delta$ may depend on $a$ but it must be bounded away from 0 when $a \rightarrow \infty$. The function $f_{a}(\zeta)$ is required to be an analytic function of the complex variable $\zeta \in H_{\delta}$ and we suppose that

$$
\frac{d^{k} f_{a}(\zeta)}{d \zeta^{k}}=O\left(|\zeta|^{\lambda_{k}} e^{\omega \zeta^{2}}\right), \quad x \rightarrow \pm \infty,
$$

where $\zeta=x+i y \in H_{\delta}$ and where $\lambda_{k}, \omega$ are real numbers not depending on $a$. Hence, the integral (1.1) converges for $a>\omega, F_{a}(-\infty)=0, F_{a}(\infty)$ is finite and these limiting values do not depend on $\operatorname{Im} \eta$, when $\eta \rightarrow \pm \infty$ in $H_{\delta}$.

The function $f_{a}(\zeta)$ can play the role of a probability density function and $F_{a}(\eta)$ can be viewed as a cumulative distribution function. Then the variable $\eta$ is related to the random variable of the underlying statistics and the parameter $a$ corresponds with the degrees of freedom. As we will show in later sections, the gamma and beta distribution functions can be transformed into (1.1). When $f_{a}(\zeta)$ is a density function, it is supposed that it is positive for real $\zeta$ and that $F_{a}(+\infty)=1$. These conditions are not required here.

From further conditions for $f_{a}(\zeta)$, which will be given in the next section, it follows that (for large a) $F_{a}(\infty)$ has an asymptotic expansion with leading term 1 . This expansion is written as

$$
F_{a}(\infty)=\sum_{s=0}^{n-1} \frac{A_{s}}{a^{s}}+\frac{\bar{A}_{n}(a)}{a^{n}}, \quad n=0,1,2, \cdots,
$$

where the coefficients $A_{s}$ do not depend on $a, A_{0}=1$ and

$$
\bar{A}_{n}(a)=O(1), \quad a \rightarrow \infty, \quad n=0,1,2, \cdots .
$$

\footnotetext{
* Received by the editors April 9, 1981.

† Stichting Mathematisch Centrum, Postbus 4079, 1009 AB Amsterdam, the Netherlands.
} 
To describe some aspects of the expansion of $F_{a}(\eta)$ we suppose that $\eta$ in (1.1) is real. For fixed values of $\eta$ we have, by using well-known methods of asymptotics,

$$
\begin{array}{ll}
\text { if } \eta<0 & F_{a}(\eta)=(2 \pi a)^{-1 / 2} e^{-a \eta^{2} / 2} \frac{f_{a}(\eta)}{-\eta}\left[1+O\left(a^{-1}\right)\right], \\
\text { if } \eta=0 & F_{a}(0)=\frac{1}{2} f_{a}(0)\left[1+O\left(a^{-1 / 2}\right)\right] \\
\text { if } \eta>0 & F_{a}(\eta)=1+O\left(a^{-1}\right) .
\end{array}
$$

From these relations it follows that the asymptotic behavior of $F_{a}(\eta)$ is completely different in the three cases distinguished and, moreover, that the asymptotic forms do not pass into one another when $\eta$ changes from negative values into positive ones. The above approximations are not uniform with respect to small values of $|\eta|$.

In asymptotics we describe these phenomena by saying that the end point of integration $\eta$ may coalesce with the saddle point at $\zeta=0$. Several contributions in the literature deal with this aspect, for instance Erdélyi (1970), Olver (1974), Bleistein and Handelsman (1975) and Wong (1980).

The object of this paper is to give an asymptotic expansion which is uniform with respect to $\eta$. The uniform approximation is not only valid in a neighborhood of $\eta=0$ but in the whole domain $H_{\delta}$. It is well known that such a uniform expansion cannot be described by elementary functions as arise in (1.5). In the present case we need the normal distribution function

$$
P(x)=(2 \pi)^{-1 / 2} \int_{-\infty}^{x} e^{-t^{2} / 2} d t
$$

This is not surprising since we know from probability theory (the central limit theorem) that the normal distribution appears if in (1.1) $a$ is large. It also follows by taking for $f_{a}(\zeta)$ a constant.

The form of the expansion for $F_{a}(\eta)$ is as follows. For $n=0,1,2, \cdots$, we write

$$
\begin{aligned}
& F_{a}(\eta)=F_{a}(\infty) P(\eta \sqrt{a})+R_{a}(\eta), \\
& R_{a}(\eta)=\frac{e^{-a \eta^{2} / 2}}{\sqrt{2 a \pi}}\left[\sum_{s=0}^{n-1} \frac{B_{s}(\eta)}{a^{s}}+\frac{\bar{B}_{n}(a, \eta)}{a^{n}}\right],
\end{aligned}
$$

where the functions $B_{s}(\eta)$ do not depend on $a$. In the next section we will give representations for $B_{s}(\eta)$ and $\bar{B}_{n}(a, \eta)$ from which information follows about the nature of the expansion in (1.7) and about the uniformity with respect to $\eta$. In $(1.7), F_{a}(\infty)$ can be replaced by (1.4).

The present paper extends the results of Temme (1979) on incomplete gamma functions to the more general class of integrals (1.1). In $\S 3$ this special case will be considered again, together with other examples. We present our results for $\eta$ in the strip $H_{\delta}$. It is possible to introduce a more general simply connected unbounded open domain $D$, which should contain each real point $\eta$ as an interior point. Depending on $f_{a}(\zeta)$, it is possible to introduce branch cuts, and $D$ is not necessarily one-sheeted. This will not be done here because it makes the presentation less transparent. However, $\mathrm{t}$ is not difficult to modify our results for such a general case, for instance, by analytic continuation. Some modifications should be made in the assumptions: $F_{a}(\infty)$ may not be finite, since it may depend on the direction in which $\eta$ approaches infinity. 


\section{Construction of the asymptotic expansion.}

2.1. Conditions on $f_{a}(\zeta)$ and $\boldsymbol{A}_{s}$. We suppose that, apart from the requirements on $f_{a}(\zeta)$ given in $\S 1, f_{a}(\zeta)$ has an asymptotic expansion

$$
f_{a}(\zeta)=\sum_{s=0}^{n-1} \frac{\phi_{s}(\zeta)}{a^{s}}+\frac{\bar{\phi}_{n}(a, \zeta)}{a^{n}}, \quad n=0,1,2, \cdots,
$$

where $\phi_{0}(0)=1$, and $\phi_{s}(\zeta)$ do not depend on $a$ and are analytic in $H_{\delta}$. For the remainders $\bar{\phi}_{n}(a, \zeta)$, we assume that

$$
\bar{\phi}_{n}(a, \zeta)=O\left(f_{a}(\zeta)\right) \text { as } a \rightarrow \infty,
$$

uniform in $H_{\delta}$ (if $f_{a}(\zeta)$ happens to vanish in $H_{\delta}$, this requirement should be modified in $\bar{\phi}_{n}(a, \zeta)=O\left(\max \left(1,\left|f_{a}(\zeta)\right|\right)\right)$.

As will be shown, it is not possible to define the expansions (1.4) and (2.1) independently of one another. There is a relation between the coefficients $A_{s}$ and the values of $\phi_{s}^{(k)}(0)$, the $k$ th derivative of $\phi_{s}(\zeta)$ at $\zeta=0$. This follows from a well-known principle in asymptotics that says that the asymptotic expansion of the integral

$$
\int_{-\infty}^{\infty} e^{-a \zeta^{2} / 2} \phi(\zeta) d \zeta, \quad a \rightarrow \infty
$$

is obtained by expanding $\phi(\zeta)$ in power of $\zeta$ and integrating term by term.

In the underlying case each term in $(2.1)$ has to be expanded. Writing

$$
\phi_{s}(\zeta)=\sum_{t=0}^{\infty} \phi_{s t} \zeta^{t}, \quad|\zeta|<\delta
$$

we obtain for $s=0,1,2,3, \cdots$ and $a \rightarrow \infty$

$$
\left(\frac{a}{2 \pi}\right)^{1 / 2} \int_{-\infty}^{\infty} e^{-a \zeta^{2} / 2} \phi_{s}(\zeta) d \zeta \sim \sum_{t=0}^{\infty} \phi_{s, 2 t}\left(\frac{2}{a}\right)^{t} \frac{\Gamma\left(t+\frac{1}{2}\right)}{\Gamma\left(\frac{1}{2}\right)} .
$$

By rearranging the results for all terms in (2.1) and by collecting terms with equal powers of $a^{-1}$, we obtain (1.4) with

$$
A_{s}=\sum_{t=0}^{s} 2^{t} \frac{\Gamma\left(t+\frac{1}{2}\right)}{\Gamma\left(\frac{1}{2}\right)} \phi_{s-t, 2 t}, \quad s=0,1,2, \cdots
$$

2.2. Integration by parts procedure. Let us first suppose that $f_{a}(\zeta)$ in (1.1) does not depend on $a$. That is, we write $\phi_{0}(\zeta)$ instead of $f_{a}(\zeta)$, and we consider

$$
F_{a}^{(0)}(\eta)=\left(\frac{a}{2 \pi}\right)^{1 / 2} \int_{-\infty}^{\eta} e^{-a \zeta^{2} / 2} \phi_{0}(\zeta) d \zeta
$$

with $\phi_{0}(0)=1$. Taking into account that the main contribution of the integral will come from a small neighborhood of the point $\zeta=0$, we write (cf. (1.6))

$$
\begin{aligned}
F_{a}^{(0)}(\eta) & =P(\eta \sqrt{a})+\left(\frac{a}{2 \pi}\right)^{1 / 2} \int_{-\infty}^{\eta} e^{-a \zeta^{2} / 2}\left[\phi_{0}(\zeta)-1\right] d \zeta \\
& =P(\eta \sqrt{a})-(2 \pi a)^{-1 / 2} \int_{-\infty}^{\eta} \frac{\phi_{0}(\zeta)-1}{\zeta} d e^{-a \zeta^{2} / 2} \\
& =P(\eta \sqrt{a})+(2 \pi a)^{-1 / 2} \frac{1-\phi_{0}(\eta)}{\eta} e^{-a \eta^{2} / 2}+(2 \pi a)^{-1 / 2} \int_{-\infty}^{\eta} \phi_{0}^{(1)}(\zeta) e^{-a \zeta^{2} / 2} d \zeta
\end{aligned}
$$


The function $\phi_{0}^{(1)}(\zeta)$ is holomorphic in $H_{\delta}$. It is given by

$$
\phi_{0}^{(1)}(\zeta)=\frac{d}{d \zeta} \frac{\phi_{0}(\zeta)-1}{\zeta}
$$

Repeating this process, we obtain

$$
F_{a}^{(0)}(\eta) \sim P(\eta \sqrt{a}) \sum_{s=0}^{\infty} \frac{A_{s}^{(0)}}{a^{s}}+\frac{e^{-a \eta^{2} / 2}}{\sqrt{2 \pi a}} \sum_{s=0}^{\infty} \frac{B_{s}^{(0)}(\eta)}{a^{s}},
$$

where $A_{s}^{(0)}$ and $B_{s}^{(0)}(\eta)$ are special cases (i.e., with $t=0$ ) of a more general set of functions defined for $t=0,1,2, \cdots$.

$$
\begin{array}{ll}
A_{s}^{(t)}=\phi_{t}^{(s)}(0), & \\
B_{s}^{(t)}(\eta)=\frac{\phi_{t}^{(s)}(0)-\phi_{t}^{(s)}(\eta)}{\eta}, & s \geqq 0 \\
\phi_{t}^{(s)}(\eta)=\frac{d}{d \eta} \frac{\phi_{t}^{(s-1)}(\eta)-\phi_{t}^{(s-1)}(0)}{\eta}, & s \geqq 1, \\
\phi_{t}^{(0)}(\eta)=\phi_{t}(\eta) . &
\end{array}
$$

The same procedure can be used for each integral

$$
F_{a}^{(t)}(\eta)=\left(\frac{a}{2 \pi}\right)^{1 / 2} \int_{-\infty}^{\eta} e^{-a \zeta^{2} / 2} \phi_{t}(\zeta) d \zeta, \quad t=0 ; 1,2, \cdots,
$$

where $\phi_{t}(\zeta)$ appears in (2.1). The result is as in (2.7), with $F_{a}^{(0)}(\eta), A_{s}^{(0)}, B_{s}^{(0)}(\eta)$ replaced by $F_{a}^{(t)}(\eta), A_{s}^{(t)}, B_{s}^{(t)}(\eta)$, respectively.

For the complete asymptotic expansion of $F_{a}(\eta)$ we collect terms of equal powers of $1 / a$ in the expansions of each $a^{-t} F_{a}^{(t)}(\eta)$, and the result is

$$
F_{a}(\eta) \sim P(\eta \sqrt{a}) \sum_{s=0}^{\infty} \frac{A_{s}}{a^{s}}+\frac{e^{-a \eta^{2} / 2}}{\sqrt{2 a \pi}} \sum_{s=0}^{\infty} \frac{B_{s}(\eta)}{a^{s}}
$$

with

$$
A_{s}=\sum_{t=0}^{s} A_{s-t}^{(t)}, \quad B_{s}(\eta)=\sum_{t=0}^{s} B_{s-t}^{(t)}(\eta) .
$$

It is not yet clear how to interpret the formal expansion (2.9) as an asymptotic expansion. In the next subsection we will discuss a method that gives a more satisfactory relation for the coefficients $A_{s}$ and $B_{s}(\eta)$ and from which a simple expression for the remainder in (2.9) follows (that is, for the function $\bar{B}_{n}(a, \eta)$ of (1.7)). The numbers $A_{s}$ constructed here are the same as those defined by (1.4) and (2.5). This is proved first.

Lemma 1. Let $A_{s}, s=0,1, \cdots$, be defined by (2.8) and (2.10). Then $A_{s}$ satisfy (2.5), with $\phi_{s t}$ given by (2.3).

Proof. The function $\phi_{t}^{(s)}(\eta)$ defined in $(2.8)$ are analytic in $H_{\delta}$. Let us define $\phi_{t r}^{(s)}$ by writing

$$
\phi_{t}^{(s)}(\eta)=\sum_{r=0}^{\infty} \phi_{t r}^{(s)} \eta^{r}, \quad|\eta|<\delta, \quad s, t=0,1,2, \cdots
$$

with $\phi_{t r}^{(0)}=\phi_{t r}$ of (2.3). By using the third line of (2.8), we obtain

$$
\phi_{t r}^{(s)}=(r+1) \phi_{t, r+2}^{(s-1)}, \quad s \geqq 1, \quad r \geqq 0, \quad t \geqq 0 .
$$


Applying this relation repeatedly, we can express $\phi_{t r}^{(s)}$ in terms of $\phi_{t r}^{(0)}=\phi_{t r}$. The result is

$$
\phi_{t r}^{(s)}=(r+1)(r+3) \cdots(r+2 s-1) \phi_{t, r+2 s}=2^{s} \frac{\Gamma\left(r / 2+\frac{1}{2}+s\right)}{\Gamma\left(r / 2+\frac{1}{2}\right)} \phi_{t, r+2 s} .
$$

$A_{s}^{(t)}$ of (2.8) satisfies $A_{s}^{(t)}=\phi_{t 0}^{(s)}$, giving for $A_{s}$ of (2.10)

$$
A_{s}=\sum_{t=0}^{s} A_{s-t}^{(t)}=\sum_{t=0}^{s} 2^{s-t} \frac{\Gamma\left(\frac{1}{2}+s-t\right)}{\Gamma\left(\frac{1}{2}\right)} \phi_{t, 2(s-t)} .
$$

This proves the lemma.

In the examples in $\S 3$, the numbers $A_{s}$ are usually obtained via (1.4). If $F_{a}(\infty)=1$, then $A_{s}=0(s \geqq 1)$, and (2.5) gives an extra relation between $\phi_{t r}$. Of course, it is possible to normalize $F_{a}(\eta)$ by dividing $f_{a}(\zeta)$ by $F_{a}(\infty)$. Then the scaled function $F_{a}(\eta)$ satisfies $F_{a}(\infty)=1$.

LEMMA 2. Let $B_{s}(\eta), s=0,1, \cdots$, be defined by (2.8) and (2.10); let the numbers $B_{\text {st }}$ be defined by

$$
B_{s}(\eta)=\sum_{t=0}^{\infty} B_{s t} \eta^{t}, \quad|\eta|<\delta
$$

Then

$$
B_{s t}=-\sum_{r=0}^{s} 2^{r} \frac{\Gamma(1+t / 2+r)}{\Gamma(1+t / 2)} \phi_{s-r, t+2 r+1} .
$$

Proof. This follows from (2.11) and the second line of (2.8)

Remark. It may be rather difficult to compute the functions $B_{s}(\eta)$ from the definitions in (2.8) and (2.10), especially for values of $\eta$ close to zero. Then the representations in (2.12) and (2.13) may be useful.

2.3. A simpler recursion for $\boldsymbol{B}_{s}(\eta)$. Consider the function $R_{a}(\eta)$ defined in the first line of (1.7). From (1.1) and (1.6) it follows that

$$
\frac{d}{d \eta} R_{a}(\eta)=(a / 2 \pi)^{1 / 2} e^{-a \eta^{2} / 2}\left[f_{a}(\eta)-F_{a}(\infty)\right] .
$$

The substitution of (1.4), (2.1) and the formal series

$$
R_{a}(\eta)=\frac{e^{-a \eta^{2} / 2}}{\sqrt{2 a \pi}} \sum_{s=0}^{\infty} \frac{B_{s}(\eta)}{a^{s}}
$$

into (2.14) shows that (2.14) is formally satisfied if

$$
\begin{aligned}
& \eta B_{0}(\eta)=1-\phi_{0}(\eta), \\
& \eta B_{s}(\eta)=A_{s}-\phi_{s}(\eta)+\frac{d}{d \eta} B_{s-1}(\eta), \quad s \geqq 1 .
\end{aligned}
$$

As outlined in foregoing subsections, the $A_{s}$ are related with the numbers $\phi_{s t}$ defined in (2.3); this relation is given in (2.5).

It is not clear, yet, that the functions $B_{s}(\eta)$ constructed in $\S 2.2$ satisfy the recursion in (2.15). This will be proved in the theorem which follows.

THEOREM 1. Let for $s=0,1,2, \cdots, B_{s}(\eta)$ be defined in (2.10) with $B_{r}^{(t)}(\eta)$ defined in (2.8). Then $B_{s}(\eta)$ satisfy the recursion relation of (2.15). 
Proof. We use induction on $s$. For $s=0$, see the second line of (2.8) with $t=0$; it gives the first relation of (2.15). Suppose, next, that the theorem is correct for the function $B_{r}(t)$ of $(2.10)$ with $0 \leqq r \leqq s-1$, for some $s \geqq 1$. Then the right-hand side of the second line of (2.15) becomes for this $s$ (see (2.10) and (2.8))

$$
\begin{aligned}
A_{s}-\phi_{s}^{(0)}(\eta)+\frac{d}{d \eta} \sum_{t=0}^{s-1} B_{s-1-t}^{(t)}(\eta) & =A_{s}-\phi_{s}(\eta)+\sum_{t=0}^{s-1} \frac{d}{d \eta} \frac{\phi_{t}^{(s-1-t)}(0)-\phi_{t}^{(s-1-t)}(\eta)}{\eta} \\
& =A_{s}-\phi_{s}(\eta)+\sum_{t=0}^{s-1} \phi_{t}^{(s-t)}(\eta)=A_{s}-\sum_{t=0}^{s} \phi_{t}^{(s-t)}(\eta) \\
& =\sum_{t=0}^{s}\left[A_{s-t}^{(t)}-\phi_{t}^{(s-t)}(\eta)\right]=\sum_{t=0}^{s}\left[\phi_{t}^{(s-t)}(0)-\phi_{t}^{(s-t)}(\eta)\right] \\
& =\eta \sum_{t=0}^{s} B_{s-t}^{(t)}(\eta)=\eta B_{s}(\eta),
\end{aligned}
$$

which proves the theorem.

\subsection{Representations for the remainder.}

THEOREM 2. Consider for (1.1) representation (1.7), where the functions $B_{s}(\eta)$ are defined in (2.15). Then for the remainder $\bar{B}_{n}(a, \eta)$ of $(1.7)$ we have for $n=0,1, \cdots$

$$
\begin{aligned}
e^{-a \eta^{2} / 2} \bar{B}_{n}(a, \eta)=-a & \int_{-\infty}^{\eta} \zeta B_{n}(\zeta) e^{-a \zeta^{2} / 2} d \zeta \\
& +\int_{-\infty}^{\eta}\left[\bar{\phi}_{n+1}(a, \zeta)-\bar{A}_{n+1}(a)\right] e^{-a \zeta^{2} / 2} d \zeta
\end{aligned}
$$

where $\bar{A}_{n}(a)$ and $\bar{\phi}_{n}(a, \zeta)$ are defined in (1.4) and (2.1).

Proof. From (2.14) (or from (1.1), (1.6), (1.7)) we have

$$
R_{a}(\eta)=\left(\frac{a}{2 \pi}\right)^{1 / 2} \int_{-\infty}^{\eta} e^{-a \zeta^{2} / 2}\left[f_{a}(\zeta)-F_{a}(\infty)\right] d \zeta
$$

Using

$$
f_{a}(\zeta)=\phi_{0}(\zeta)+\frac{\bar{\phi}_{1}(a, \zeta)}{a}, \quad F_{a}(\infty)=1+\frac{\bar{A}_{1}(a)}{a}, \quad R_{a}(\eta)=\frac{e^{-a \eta^{2} / 2}}{\sqrt{2 a \pi}} \bar{B}_{0}(a, \eta),
$$

we obtain

$$
\begin{aligned}
R_{a}(\eta) & =\left(\frac{a}{2 \pi}\right)^{1 / 2} \int_{-\infty}^{\eta} e^{-a \zeta^{2} / 2}\left[\phi_{0}(\zeta)-1+\frac{\bar{\phi}_{1}(a, \zeta)}{a}-\frac{\bar{A}_{1}(a)}{a}\right] d \zeta \\
& =(2 \pi a)^{-1 / 2} \int_{-\infty}^{\eta} e^{-a \zeta^{2} / 2}\left[-a \zeta B_{0}(\zeta)+\bar{\phi}_{1}(a, \zeta)-\bar{A}_{1}(a)\right] d \zeta
\end{aligned}
$$

which gives (2.16) with $n=0$. Considering (2.16) with $n \geqq 0$ we proceed by using the obvious relations

$$
\begin{aligned}
& \bar{\phi}_{n+1}(a, \zeta)=\phi_{n+1}(\zeta)+\frac{\bar{\phi}_{n+2}(a, \zeta)}{a}, \\
& \bar{A}_{n+1}(a)=A_{n+1}+\frac{\bar{A}_{n+2}(a)}{a}, \\
& \bar{B}_{n}(a, \eta)=B_{n}(\eta)+\frac{\bar{B}_{n+1}(a, \eta)}{a} .
\end{aligned}
$$


Then, by partial integration of the first integral in (2.16), this formula becomes

$$
\begin{aligned}
e^{-a \eta^{2} / 2}\left[B_{n}(\eta)+\frac{\bar{B}_{n+1}(a, \eta)}{a}\right] \\
=\left.e^{-a \zeta^{2} / 2} B_{n}(\zeta)\right|_{\zeta=-\infty} ^{\zeta=\eta}+\int_{-\infty}^{\eta}\left[\phi_{n+1}(\zeta)-A_{n+1}-\frac{d}{d \zeta} B_{n}(\zeta)+\frac{\bar{\phi}_{n+2}(a, \zeta)}{a}-\frac{\bar{A}_{n+2}(a)}{a}\right] d \zeta .
\end{aligned}
$$

The integrated term vanishes at $\zeta=-\infty$ (this follows from (1.3)), and by using (2.15) we obtain (2.16) with $n$ replaced by $n+1$.

THEOREM 3. Under the same conditions as in Theorem 2 we have for $n=$ $0,1,2, \cdots$

$$
e^{-a \eta^{2} / 2} \bar{B}_{n}(a, \eta)=a \int_{\eta}^{\infty} \zeta B_{n}(\zeta) e^{-a \zeta^{2} / 2} d \zeta+\int_{\eta}^{\infty}\left[\bar{A}_{n+1}(a)-\bar{\phi}_{n+1}(a, \zeta)\right] e^{-a \zeta^{2} / 2} d \zeta
$$

Proof. From the definition of $R_{a}(\eta)$ in (1.7) it follows that

$$
\lim _{x \rightarrow \pm \infty} R_{a}(\eta)=0, \quad \eta=x+i y \in H_{\delta}
$$

Hence, we could have started with (2.17) with interval of integration $[\eta, \infty)$ and a different sign before the integral. The rest of the proof runs as in Theorem 2 .

It follows from both theorems that, for fixed real values of $\eta, \bar{B}_{n}(a, \eta)$ of (1.7) satisfy

$$
\bar{B}_{n}(a, \eta)=O(1), \quad a \rightarrow \infty .
$$

For $\eta \leqq 0$ this follows from (2.16), for $\eta \geqq 0$ from (2.18). Hence, again for fixed $\eta$, $R_{a}(\eta)$ of (1.7) satisfy

$$
R_{a}(\eta)=O\left(a^{-1 / 2} e^{-a \eta^{2} / 2}\right), \quad a \rightarrow \infty .
$$

It is not difficult to show that (2.19) and (2.20) hold uniformly with respect to $\eta$ in compact subsets of $H_{\delta}$. In all relevant applications (see $\S 3$ ) the number $\omega$ of $(1.3)$ is 0 . Also, bounds for $\bar{B}_{n}(a, \eta)$ can be constructed that are holding uniformly with respect to $\eta \in H_{\delta}$.

In our previous paper Temme (1979) on incomplete gamma functions such bounds for $\ddot{B}_{n}(a, \eta)$ were computed for $n=0,1, \cdots, 10$. In that paper we overlooked the remarkable point that both representations $(2.16)$ and $(2.18)$ can be used for constructing these bounds. Consequently, formula (2.17) of that paper should be revised for $\eta<0$ and (2.19) for $\eta>0$. Also, Remark 1 in the cited reference (p. 760) should be skipped. More details will be given in $\S 3.4$.

In the next section some examples are worked out. In each example the functions are related with statistical distribution functions. Usually transformations are needed for a representation in the standard form (1.1).

\section{Examples.}

3.1. Incomplete normal distribution function. We consider

$$
F_{a}(\eta)=\left(\frac{a}{2 \pi}\right)^{1 / 2} \int_{-\infty}^{\eta} e^{-a \zeta^{2} / 2} \frac{d \zeta}{1+\zeta^{2}}
$$

in the strip $H_{\delta}$ with $\delta=1$. This function finds wide application in probability theory, mathematical statistics and in problems involving the heat conduction equation. Jones 
(1972) used it for describing the asymptotic expansion of a double integral. It constitutes a sort of generalization of the error integral. To see this note that

$$
F_{a}(\infty)=(2 a \pi)^{1 / 2} e^{a / 2} Q(\sqrt{a}),
$$

where $Q(x)=1-P(x)=\frac{1}{2} \operatorname{erfc}(x / \sqrt{2})$.

The function $f_{a}(\zeta)$ of $(1.1)$ is here $1 /\left(1+\zeta^{2}\right)$, and in this simple case the coefficients $B_{s}(\eta)$ can be constructed rather easily. We have $f_{a}(\zeta)=\phi_{0}(\zeta)$ and $\phi_{s}(\zeta)=0(s \geqq 1)$. From the known expansion of the error function we obtain

$$
F_{a}(\infty) \sim \sum_{s=0}^{\infty} \frac{A_{s}}{a^{s}}, \quad A_{0}=1, \quad A_{s}=\frac{(-2)^{s} \Gamma\left(s+\frac{1}{2}\right)}{\Gamma\left(\frac{1}{2}\right) .}, \quad s \geqq 1,
$$

which can also be obtained from (2.5) with

$$
\phi_{s t}= \begin{cases}0 & \text { if } s \geqq 1 \text { or } t=2 r+1, \quad r \geqq 0, \\ (-1)^{t / 2} & \text { else. }\end{cases}
$$

Hence, from (2.12), (2.13) it follows that for $|\eta|<1$

$$
B_{s}(\eta)=\eta(-2)^{s} \sum_{t=0}^{\infty} \frac{\Gamma\left(t+s+\frac{3}{2}\right)}{\Gamma\left(t+\frac{3}{2}\right)}\left(-\eta^{2}\right)^{t},
$$

which can be expressed in terms of Gauss' hypergeometric function ${ }_{2} F_{1}(a, b ; c ; z)$ :

$$
\begin{aligned}
B_{s}(\eta) & =\eta(-2)^{s} \frac{\Gamma\left(s+\frac{3}{2}\right)}{\Gamma\left(\frac{3}{2}\right)}{ }_{2} F_{1}\left(s+\frac{3}{2}, 1 ; \frac{3}{2} ;-\eta^{2}\right) \\
& =\eta(-2)^{s} \frac{\Gamma\left(s+\frac{3}{2}\right)}{\Gamma\left(\frac{3}{2}\right)}\left(1+\eta^{2}\right)^{-1}{ }_{2} F_{1}\left(1,-s ; \frac{3}{2} ; \frac{\eta^{2}}{\eta^{2}+1}\right) .
\end{aligned}
$$

The second relation enables us to write

$$
B_{s}(\eta)=\frac{1}{2} \eta(-2)^{s} \frac{\Gamma\left(s+\frac{3}{2}\right)}{\left(1+\eta^{2}\right) \Gamma\left(\frac{3}{2}\right)} \int_{0}^{1}(1-t)^{-1 / 2}\left(1-t \frac{\eta^{2}}{\eta^{2}+1}\right)^{s} d t,
$$

from which we obtain

$$
\left|B_{s}(\eta)\right| \leqq \frac{|\eta|}{1+\eta^{2}} 2^{s} \frac{\Gamma\left(s+\frac{3}{2}\right)}{\Gamma\left(\frac{3}{2}\right)}, \quad \eta \in \mathbb{R} .
$$

Hence, $B_{s}(\eta)$ are bounded on $\mathbb{R}$.

The representation for (3.1) becomes (see (1.7))

$$
F_{a}(\eta)=F_{a}(\infty) P(\eta \sqrt{a})+\frac{e^{-a \eta^{2} / 2}}{\sqrt{2 a \pi}}\left[\sum_{s=0}^{n-1} \frac{B_{s}(\eta)}{a^{s}}+\frac{\bar{B}_{n}(a, \eta)}{a^{n}}\right],
$$

with $B_{0}(\eta)=\eta /\left(1+\eta^{2}\right), B_{1}(\eta)=-\eta\left(3+\eta^{2}\right) /\left(1+\eta^{2}\right)^{2}$. In this example other $B_{s}(\eta)$ can be computed by using recursion relations of ${ }_{2} F_{1}$-functions. It easily follows that

$$
\left(1+\eta^{2}\right) B_{s+1}(\eta)=-2 s(2 s+1) B_{s-1}(\eta)-\left[(2 s+1) \eta^{2}+4 s+3\right] B_{s}(\eta) .
$$

To estimate the remainder we remark that in $(2.16) \bar{\phi}_{n+1}(a, \zeta)=0$ and $\left|\bar{A}_{n+1}(a)\right|$ can be replaced by $A_{n+1}$. This follows from a known result about the asymptotic expansion of the error function

$$
F_{a}(\infty)=\left(\frac{a \pi}{2}\right)^{1 / 2} e^{a / 2} \operatorname{erfc}\left(\frac{a}{2}\right)^{1 / 2}=\sum_{s=0}^{n-1} \frac{A_{s}}{a^{s}}+\frac{\overline{A_{n}}(a)}{a^{n}},
$$

where $\bar{A}_{n}(a)=\theta_{n} A_{n}, n=0,1, \cdots$ with $0<\theta_{n}<1$. 
It follows that (see $(2.16)$ )

$$
\left|e^{-a \eta^{2} / 2} \bar{B}_{n}(a, \eta)\right| \leqq \frac{2^{n} \Gamma\left(n+\frac{3}{2}\right)}{\Gamma\left(\frac{3}{2}\right)} \int_{-\infty}^{\eta} \frac{1+a \zeta^{2}}{1+\zeta^{2}} e^{-a \zeta^{2} / 2} d \zeta .
$$

The integral can be expressed in terms of $F_{a}(\eta)$ and $P(\eta \sqrt{a})$. This estimate is valid for $\eta \in \mathbb{R}$. However, for $\eta \geqq 0$ it is better to use the representation of (2.18) which gives the same expression with an interval of integration $[\eta, \infty)$.

It follows that (3.2) can be used throughout the strip $H_{1}$. It is not difficult to extend the results for a larger domain, or for complex values of $a$.

3.2. Sievert integral. This integral is defined as

$$
I(\theta, a)=\int_{-\pi / 2}^{\theta} e^{a(1-1 / \cos \phi)} d \phi,
$$

$a>0,-\pi / 2<\theta<\pi / 2$. The complete integral $I(\pi / 2, a)$ is an integral of the modified Bessel function $K_{0}(x)$, that is,

$$
I(\pi / 2, a)=2 e^{a} \int_{a}^{\infty} K_{0}(x) d x .
$$

We need a transformation in order to bring (3.3) into the standard form (1.1). The appropriate change of variables is defined by

$$
\begin{array}{ll}
-\frac{1}{2} \zeta^{2}=1-1 / \cos \phi, & \zeta=\frac{2 \sin (\phi / 2)}{\sqrt{\cos \phi}}, \\
-\frac{1}{2} \eta^{2}=1-1 / \cos \theta, & \eta=\frac{2 \sin (\theta / 2)}{\sqrt{\cos \theta}},
\end{array}
$$

and the integral (3.2) becomes

$$
I(\theta, a)=\int_{-\infty}^{n} e^{-a \zeta^{2} / 2} \frac{d \phi}{d \zeta} d \zeta
$$

where $\mathrm{d} \phi / \mathrm{d} \zeta=\left[\left(1+\zeta^{2} / 2\right)\left(1+\zeta^{2} / 4\right)^{1 / 2}\right]^{-1}$, which is holomorphic in a strip $H_{\delta}$ in the $\zeta$-plane with $\delta=\sqrt{2}$.

Remark. It is important to note that the mapping (3.5) of the $\phi$-interval into the $\zeta$-interval involves a square root $\zeta=2\left[\sin ^{2}(\phi / 2) / \cos \phi\right]^{1 / 2}$, where the sign of the square root has the sign of $\phi$. In this way $\zeta$ becomes a holomorphic function of $\phi$ at $\phi=0$. The same for $\eta$ and $\theta$.

The standard form (1.1) is now achieved by writing

$$
F_{a}(\eta)=\left(\frac{a}{2 \pi}\right)^{1 / 2} I(\theta, a)=\left(\frac{a}{2 \pi}\right)^{1 / 2} \int_{-\infty}^{\eta} e^{-a \zeta^{2} / 2} f_{a}(\zeta) d \zeta, \quad f_{a}(\zeta)=\frac{d \phi}{d \zeta}
$$

and $F_{a}(\infty)$ has the known expansion

$$
\begin{aligned}
& F_{a}(\infty)=2\left(\frac{a}{2 \pi}\right)^{1 / 2} e^{a} \int_{a}^{\infty} K_{0}(x) d x \sim \sum_{n=0}^{\infty} \frac{A_{s}}{a^{s}}, \\
& A_{s}=(-1)^{s} \frac{\Gamma\left(s+\frac{1}{2}\right)}{\Gamma\left(\frac{1}{2}\right)} \sum_{t=0}^{s} 2^{-t} \frac{\Gamma\left(t+\frac{1}{2}\right)}{2 t ! \Gamma\left(\frac{1}{2}\right)} .
\end{aligned}
$$


The asymptotic expansion as in (1.7) can now be given for $a \rightarrow \infty$, uniform with respect to $\eta \in H_{\delta}(\delta=\sqrt{2})$. The relation between $\eta$ and the original variable $\theta$ is given in (3.5). Complex values of $\eta$ correspond to complex values of $\theta$. In fact, (3.5) defines a conformal mapping between parts of the complex $\eta$ and $\theta$ planes, and the asymptotic expansion is valid for complex $\theta$ in the image of $H_{\delta}$ under this mapping. As in the foregoing example, the coefficients $B_{s}(\eta)$ can be expressed in terms of hypergeometric functions.

The expansion reads

$$
\begin{aligned}
& \left(\frac{a}{2 \pi}\right)^{1 / 2} I(\theta, a) \sim F_{a}(\infty) P\left(2 \sin \frac{1}{2} \theta \sqrt{a / \cos \theta}\right)+\frac{e^{a(1-1 / \cos \theta)}}{\sqrt{2 \pi a}} \sum_{s=0}^{\infty} \frac{B_{s}(\eta)}{a^{s}} \\
& B_{0}(\eta)=\frac{\cos (\theta / 2) \sqrt{\cos \theta}-\cos ^{2} \theta}{\sin \theta} .
\end{aligned}
$$

3.3. Incomplete beta function. This well-known function is given by

$$
I_{x}(p, q)=\frac{1}{B(p, q)} \int_{0}^{x} t^{p-1}(1-t)^{q-1} d t .
$$

We consider $0 \leqq x \leqq 1, p>0, q>0$; however, extension to complex values is possible. $B(p, q)$ is Euler's (complete) beta function

$$
B(p, q)=\int_{0}^{1} t^{p-1}(1-t)^{q-1} d t=\frac{\Gamma(p) \Gamma(q)}{\Gamma(p+q)}
$$

We consider the asymptotic expansion of $I_{x}(p, q)$ for large $p$ (or $q$ or both) uniformly with respect to $x \in[0,1]$. Since

$$
I_{x}(p, q)=1-I_{1-x}(q, p),
$$

we can consider $p \geqq q$.

The incomplete beta function is a standard probability function. As special cases it has the (negative) binomial distribution, Student's distribution and the $F$-(varianceratio) distribution.

We start with the symmetric case $p=q$, which is rather easy to handle compared with the general situation.

3.3.1. The symmetric case $p=q=a$. A trivial transformation gives

$$
I_{x}(p, q)=\frac{4^{-a}}{B(a, a)} \int_{0}^{x} e^{a \ln [4 t(1-t)]} \frac{d t}{t(1-t)} .
$$

The next appropriate transformations are

$$
\begin{aligned}
& -\frac{1}{2} \zeta^{2}=\ln [4 t(1-t)], \quad 0<t<1, \quad \operatorname{sign}(\zeta)=\operatorname{sign}\left(t-\frac{1}{2}\right), \\
& -\frac{1}{2} \eta^{2}=\ln [4 x(1-x)], \quad 0<x<1, \quad \operatorname{sign}(\eta)=\operatorname{sign}\left(x-\frac{1}{2}\right) \text {. }
\end{aligned}
$$


Then we have the standard form (1.1), with

$$
\begin{aligned}
& F_{a}(\eta)=I_{x}(a, a)=\left(\frac{a}{2 \pi}\right)^{1 / 2} \int_{-\infty}^{\eta} e^{-a \zeta^{2} / 2} f_{a}(\zeta) d \zeta, \\
& f_{a}(\zeta)=\frac{4^{-a}}{B(a, a)}\left(\frac{\pi}{a}\right)^{1 / 2}\left[\frac{1}{2} \zeta^{2} /\left(1-\mathrm{e}^{-\zeta^{2} / 2}\right)\right]^{1 / 2}
\end{aligned}
$$

where the square root is positive for real values of its argument. Considered as a function of the complex variable $\zeta, f_{a}(\zeta)$ is holomorphic in $H_{\delta}$ with $\delta=\sqrt{2 \pi}$. By writing

$$
\frac{4^{-a}(\pi / a)^{1 / 2}}{B(a, a)}=\frac{a^{-1 / 2} \Gamma\left(a+\frac{1}{2}\right)}{\Gamma(a)} \sim \sum_{s=0}^{\infty} c_{s} a^{-s}, \quad a \rightarrow \infty,
$$

where $c_{s}$ can be expressed in terms of Bernoulli polynomials (see Luke (1969, vol. I, p. 33)), it follows that

$$
\begin{gathered}
f_{a}(\zeta) \sim \phi_{0}(\zeta) \sum_{s=0}^{\infty} \frac{c_{s}}{a^{s}}, \quad a \rightarrow \infty, \\
\phi_{0}(\zeta)=\left[\frac{1}{2} \zeta^{2} /\left(1-\mathrm{e}^{-\zeta^{2} / 2}\right)\right]^{1 / 2}=\sum_{s=0}^{\infty} \frac{B_{s}^{(1 / 2)}\left(\frac{1}{2}\right)}{s !}\left(\frac{1}{2} \zeta^{2}\right)^{s} .
\end{gathered}
$$

The coefficients in this expansion are generalized Bernoulli polynomials; see again Luke (1969, vol. I, p. 18). In this case $F_{a}(\infty)=1$; hence, $A_{0}=1, A_{s}=0(s \geqq 1)$. A further analysis shows that all $B_{s}(\eta)$ are bounded functions of $\eta$ on $(-\infty, \infty)$.

3.3.2. The general case $p \geqq q$. Let us write in (3.10)

$$
p=a \sin ^{2} \theta, \quad q=a \cos ^{2} \theta, \quad 0 \leqq \theta \leqq \frac{\pi}{2} .
$$

Then

$$
I_{x}(p, q)=\frac{1}{B(p, q)} \int_{0}^{x} \exp \left(a\left(\sin ^{2} \theta \ln t+\cos ^{2} \theta \ln (1-t)\right) \frac{d t}{t(1-t)} .\right.
$$

The maximum of the integrand occurs at $t=\sin ^{2} \theta$, Hence, the transformation $t \rightarrow \zeta$ reads

$$
-\frac{1}{2} \zeta^{2}=\sin ^{2} \theta \ln \frac{t}{\sin ^{2} \theta}+\cos ^{2} \theta \ln \frac{1-t}{\cos ^{2} \theta},
$$

where the sign of $\zeta$ equals the sign of $t-\sin ^{2} \theta$ (for real variables, for complex variables it is defined by analytic continuation of the real case). A similar transformation holds for $x \rightarrow \eta$ if $t$ and $\zeta$ are replaced by $x$ and $\eta$, respectively. From (3.14) we obtain

$$
-\zeta \frac{d \zeta}{d t}=\frac{\sin ^{2} \theta-t}{t(1-t)}
$$

hence the representation of (3.13) in the standard form is

$$
\begin{aligned}
& F_{a}(\eta)=I_{x}(p, q)=\left(\frac{a}{2 \pi}\right)^{1 / 2} \int_{-\infty}^{\eta} e^{-a \zeta^{2} / 2} f_{a}(\zeta) d \zeta, \\
& f_{a}(\zeta)=\left(\frac{a}{2 \pi}\right)^{-1 / 2} \frac{\exp \left[a\left(\sin ^{2} \theta \ln \sin ^{2} \theta+\cos ^{2} \theta \ln \cos ^{2} \theta\right)\right]}{B(p, q) \sin \theta \cos \theta} \frac{\zeta \cos \theta \sin \theta}{t-\sin ^{2} \theta} .
\end{aligned}
$$


It follows, and this is well known, that the critical point $\eta=0$ corresponds with $x=\sin ^{2} \theta=p /(p+q)$. That is, when for large $a=p+q$ the parameter $x$ crosses the value $p /(p+q)$, the function $I_{x}(p, q)$ changes suddenly from values close to zero into values close to 1 .

It remains to show that $f_{a}(\zeta)$ of $(3.15)$ has the properties as supposed in $\S \S 1$ and 2 . First we consider the $\zeta$-part of it which is defined by

$$
\phi_{0}(\zeta)=\frac{\zeta \cos \theta \sin \theta}{t-\sin ^{2} \theta}
$$

where $\zeta$ and $t(\zeta)$ are related by (3.14); $t=\sin ^{2} \theta$ corresponds with $\zeta=0$. At this point $\phi_{0}(\zeta)$ is regular and $\phi_{0}(0)=1$. Due to the many-valuedness of the logarithms in (3.14), we have other finite singularities in the $\zeta$-plane. These singularities occur for $t=$ $\sin ^{2} \theta \exp (2 \pi i n)$ or $(1-t)=\cos ^{2} \theta \exp (2 \pi i m), n, m= \pm 1, \pm 2, \cdots$ in the manysheeted $t$-plane. Corresponding $\zeta$-values follow from (3.14)

$$
-\frac{1}{2} \zeta_{n}^{2}=2 \pi i n \sin ^{2} \theta, \quad-\frac{1}{2} \zeta_{m}^{2}=2 \pi i m \cos ^{2} \theta
$$

These $\zeta$-values have imaginary parts

$$
\pm \sin \theta \sqrt{2 \pi n}, \quad \pm \cos \theta \sqrt{2 \pi m} \text {. }
$$

If $p \geqq q$, we have $\sin \theta \geqq \cos \theta$. Hence, $\phi_{0}(\zeta)$ is holomorphic in $H_{\delta}$ with $\delta=\cos \theta \sqrt{2 \pi}$, with $\cos \theta=\sqrt{q /(p+q)}$. It follows that $\delta \rightarrow 0$, if $q /(p+q) \rightarrow 0$ for large $a=p+q$. We can apply the methods of $\S 2$ if $\delta$ is bounded away from zero. An important conclusion is that, when $p \rightarrow \infty, q$ should also grow. Otherwise the strip $H_{\delta}$ will coincide with $\mathbb{R}$ since the singularities approach the origin $\zeta=0$ (the saddle point).

We proceed by supposing that $p \rightarrow \infty, q \rightarrow \infty$, such that $q /(p+q) \geqq \varepsilon>0$, where $\varepsilon$ does not depend on $p$ or $q$.

The remaining part of (3.15), that is, the part not including (3.16), can be expanded in terms of the large parameter $a=p+q$. By using the well-known Stirling approximations of the gamma function, we obtain

$$
f_{a}(\zeta) \sim \phi_{0}(\zeta) \sum_{s=0}^{\infty} \frac{c_{s}(\theta)}{a^{s}}, \quad a \rightarrow \infty,
$$

with $\left.c_{0}(\theta)=1, c_{1}(\theta)=\frac{1}{12}\left[1-1 / \sin ^{2} \theta \cos ^{2} \theta\right)\right]$. Hence, the function $f_{a}(\zeta)$ satisfies the requirements of $\S \S 1$ and $2 ; F_{a}(\infty)=1$, and we can compute the functions $B_{s}(\eta)(s \geqq 1)$ from

$$
B_{0}(\eta)=\frac{1-\phi_{0}(\eta)}{\eta}
$$

with $\phi_{0}(\eta)$ given in (3.16). The expansion

$$
F_{a}(\eta)=I_{x}(p, q) \sim P(\eta \sqrt{a})+\frac{e^{-a \eta^{2} / 2}}{\sqrt{2 \pi a}} \sum_{s=0}^{\infty} \frac{B_{s}(\eta)}{a^{s}},
$$

$a=p+q \rightarrow \infty$, is uniformly valid with respect to $x \in[0,1]$ as long as $q /(p+q) \geqq \varepsilon>0$ (for the case $p \geqq q$; if $q \geqq p$, we suppose that $p /(p+q) \geqq \varepsilon>0$ ).

It is not surprising that we cannot obtain a uniform expansion in this way. The problem is to find an expansion for $a=p+q \rightarrow \infty$, which is uniform with respect to both $x$ and $\theta$, with $x \in[0,1], \theta \in[0, \pi / 2]$. Thus there is one asymptotic variable $(a)$ and two nonasymptotic variables $(x$ and $\theta$ ). It is expected that to cover the whole domain of interest in the $x \times \theta$ space, it will be necessary to resort to a transcendental function of two variables as approximant (see Olver (1975)). 
It is possible to treat the case $p \rightarrow \infty, q$ fixed, by using a method given by Erdélyi (1974) (see also Temme (1976)). Let us write (3.10) in the form

$$
I_{x}(p, q)=\frac{1}{B(p, q)} \int_{-\ln x}^{\infty} e^{-p t} t^{q-1}\left[\frac{1-e^{-t}}{t}\right]^{q-1} d t
$$

which satisfies (by adapting the notation) the requirements for obtaining the asymptotic expansion for $p \rightarrow \infty$, uniformly valid in $x \in[0,1] ; q$ is a fixed parameter. We restrict $q$ to the interval $(0,1)$, but there is no loss of generality in this assumption. Erdélyi's expansion is of the form

$$
I_{x}(p, q) \sim \frac{1}{B(p, q)}\left[p^{-q} Q(q,-p \ln x) \sum_{s=0}^{\infty} \frac{A_{s}(q)}{p^{s}}+x^{p} \sum_{s=1}^{\infty} \frac{B_{s}(x, q)}{p^{s}}\right],
$$

where $Q(a, z)$ is an incomplete gamma function (see next example). The construction of the coefficients $A_{s}(q)$ and $B_{s}(x, q)$ is outlined in Erdélyi's paper. Note that $Q(a, z)$ is a function of two variables. It is still an open problem how to modify (3.17) and (3.18) in order to obtain an expansion for $I_{x}(p, q)$ for $p \rightarrow \infty$, uniformly in $x \in[0,1]$ and $q \geqq \varepsilon>0$.

3.4. Incomplete gamma functions. This important example is considered earlier in Temme (1979). The present method gives the same asymptotic expansion. The incomplete gamma functions are

$$
P(a, x)=\frac{1}{\Gamma(a)} \int_{0}^{x} t^{a-1} e^{-t} d t, \quad Q(a, x)=\frac{1}{\Gamma(a)} \int_{x}^{\infty} t^{a-1} e^{-t} d t .
$$

The basic transformations for the integrals are applied on

$$
P(a, x)=\frac{e^{-a} a^{a}}{\Gamma(a)} \int_{0}^{x / a} e^{-a(-\ln t+t-1)} t^{-1} d t
$$

by defining

$$
\begin{aligned}
& \frac{1}{2} \zeta^{2}=-\ln t+t-1, \quad \operatorname{sign} \zeta=\operatorname{sign}(t-1) \\
& \frac{1}{2} \eta^{2}=-\ln \lambda+\lambda-1, \quad \operatorname{sign} \eta=\operatorname{sign}(\lambda-1), \quad \lambda=\frac{x}{a} .
\end{aligned}
$$

The result is

$$
\begin{aligned}
& F_{a}(\eta)=P(a, x)=\left(\frac{a}{2 \pi}\right)^{1 / 2} \int_{-\infty}^{\eta} e^{-a \zeta^{2} / 2} f_{a}(\zeta) d \zeta, \\
& G_{a}(\eta)=Q(a, x)=\left(\frac{a}{2 \pi}\right)^{1 / 2} \int_{\eta}^{\infty} e^{-a \zeta^{2} / 2} f_{a}(\zeta) d \zeta
\end{aligned}
$$

with

$$
f_{a}(\zeta)=\frac{e^{-a} a^{a}(2 \pi / a)^{1 / 2}}{\Gamma(a)} \frac{\zeta}{t-1} \sim \phi_{0}(\zeta) \sum_{s=0}^{\infty} \frac{\gamma_{s}}{a^{s}}, \quad \phi_{0}(\zeta)=\frac{\zeta}{t-1},
$$

and the numbers $\gamma_{s}$ are the well-known coefficients appearing in the expansion of the reciprocal gamma function. For more details we refer to Temme (1979). 
The expansions are (note that $F_{a}(\infty)=1$ )

$$
\begin{aligned}
& P(a, x)=P(\eta \sqrt{a})+\frac{e^{-a \eta^{2} / 2}}{\sqrt{2 \pi a}}\left[\sum_{s=0}^{n-1} \frac{B_{s}(\eta)}{a^{s}}+\frac{\bar{B}_{n}(a, \eta)}{a^{n}}\right], \\
& Q(a, x)=Q(\eta \sqrt{a})-\frac{e^{-a \eta^{2} / 2}}{\sqrt{2 \pi a}}\left[\sum_{s=0}^{n-1} \frac{B_{s}(\eta)}{a^{s}}+\frac{\bar{B}_{n}(a, \eta)}{a^{n}}\right], \\
& B_{0}(\eta)=\frac{1}{\eta}-\frac{1}{\lambda-1}, B_{1}(\eta)=\frac{1}{(\lambda-1)^{3}}+\frac{1}{(\lambda-1)^{2}}+\frac{1}{12(\lambda-1)}-\frac{1}{\eta^{3}} .
\end{aligned}
$$

$B_{s}(\eta)$ are the same as $-c_{s}(\eta)$ in our previous paper.

As mentioned in $\S 2.4$, the bounds given there can be sharpened. By using Theorems 2 and 3, we obtain

$$
\left|\bar{B}_{n}(a, \eta)\right| \leqq(\lambda+1)^{-\kappa} C_{n} e^{-a \eta^{2} / 2}+\left|H_{n+1}(a)\right| e^{a} a^{-a} \Gamma(a) D(a, x),
$$

where $\kappa=\frac{1}{2}$ for $n=0,1$ for $n \geqq 1$,

$$
D(a, x)=\min (P(a, x), Q(a, x)), \quad C_{s}=\sup _{\eta \in \mathbb{R}}\left[(1+\lambda)^{\kappa}\left|B_{s}(\eta)\right|\right], \quad s=0,1, \ldots
$$

and $H_{n}(a)$ is the remainder in

$$
\frac{1}{\Gamma(a)}=e^{a} a^{-a}\left(\frac{a}{2 \pi}\right)^{1 / 2}\left[\sum_{s=0}^{n-1} \frac{\gamma_{s}}{a^{s}}+\frac{H_{n}(a)}{a^{n}}\right] .
$$

The numbers $C_{n}$ and bounds for $H_{n}(a)$ are given in Temme (1979).

3.5. Pearson type IV probability function. We consider it in the form (see Fettis (1976))

$$
I(\theta, \alpha, \beta)=\int_{-\pi / 2}^{\theta} e^{2 \beta \phi} \cos ^{2 \alpha} \phi d \phi,
$$

for large positive values of $\alpha ;-\pi / 2<\theta<\pi / 2 ; \beta$ is a real parameter. It is known that

$$
I\left(\frac{1}{2} \pi, \alpha, \beta\right)=\frac{\pi \Gamma(1+2 \alpha) 2^{-2 \alpha}}{\Gamma(1+\alpha+i \beta) \Gamma(1+\alpha-i \beta)} .
$$

The maximum of the integrand occurs at $\phi=\lambda:=\arctan \gamma$, with $\gamma=\beta / \alpha$. The appropriate transformations are

$$
-\frac{1}{2} \zeta^{2}=\gamma(\phi-\lambda)+\ln \frac{\cos \phi}{\cos \lambda}, \quad-\frac{1}{2} \eta^{2}=\gamma(\theta-\lambda)+\ln \frac{\cos \theta}{\cos \lambda},
$$

with $\operatorname{sign} \zeta=\operatorname{sign}(\phi-\lambda)$, sign $\eta=\operatorname{sign}(\theta-\lambda)$. Hence, with $a=2 \alpha$, we obtain

$$
F_{a}(\eta)=\frac{I(\theta, \alpha, \beta)}{I(\pi / 2, \alpha, \beta)}=\left(\frac{a}{2 \pi}\right)^{1 / 2} \int_{-\infty}^{\eta} e^{-a \zeta^{2} / 2} f_{a}(\zeta) d \zeta,
$$

with

$$
\begin{aligned}
& f_{a}(\zeta)=\frac{e^{a(\gamma \lambda+\ln \cos \lambda)}}{I(\pi / 2, \alpha, \beta)}\left(\frac{2 \pi}{a}\right)^{1 / 2} \frac{d \phi}{d \zeta} \sim \phi_{0}(\zeta) \sum_{s=0}^{\infty} \frac{c_{s}(\gamma)}{a^{s}} \\
& \phi_{0}(\zeta)=\frac{1}{\cos \lambda} \frac{\zeta}{\tan \phi-\gamma}, \quad \phi_{0}(0)=1 .
\end{aligned}
$$




\section{REFERENCES}

N. Bleistein AND R. A. HANDElsman (1975). Asymptotic Expansion of Integrals, Holt, Rinehart and Winston, New York.

A. ERDÉLYI (1970), Uniform asymptotic expansion of integrals, in Analytical Methods in Mathematical Physics, R. P. Gilbert, R. G. Newton, eds, Gordon and Breach, New York.

(1974), Asymptotic evaluation of integrals involving a fractional derivative, this Journal, 5, pp. 159-171.

H. E. FeTtIS, (1976), Fourier series expansions for Pearson type IV distributions and probabilities, SIAM J. Appl. Math. 31, pp. 511-518.

D. S. JoneS, (1972), Asymptotic behaviour of an aperture integral, Proc. Roy. Soc. Edinburgh (A), 71, pp. 9-29.

Y. L. Luke, (1969), The Special Functions and Their Approximations, Academic Press, New York.

F. W. J. Olver, (1974), Asymptotics and Special Functions, Academic Press, New York.

(1975), Unsolved problems in the asymptotic estimation of special functions, in Theory and Applications of Special Functions, R. Askey, ed., Academic Press, New York.

N. M. TEMME, (1976), Remarks on a paper of A. Erdélyi, this Journal, 7, pp. 767-770.

, (1979), The asymptotic expansions of the incomplete gamma functions, this Journal, 10, pp. 757-766.

R. WONG. (1980), Error bounds for asymptotic expansions of integrals, SIAM Rev., 22, pp. 401-435. 